\title{
Discrete Cosine Transform and Support Vector Machines for Classification Cardiac Atrial Arrhythmia and Cardiac Normal
}

\author{
Ratnadewi $^{1 *}$, AanDarmawanHangkawidjaja ${ }^{1}$, Agus Prijono $^{1}$, Jo Suherman $^{2}$ \\ ${ }^{1}$ Faculty of Engineering, UniversitasKristen Maranatha,Bandung, Indonesia \\ ${ }^{2}$ Faculty of Medicine, Universitas Kristen Maranatha, Bandung, Indonesia \\ ratnadewi.bandung@gmail.com; ratnadewi@maranatha.ac.id
}

\begin{abstract}
The electrocardiogram signal is the most important analysis to detect cardiac arrhythmia. Machine learning classification is used as a first step to detect someone's arrhythmia or normal heart. This paper discusses one method for detecting arrhythmia by using digital images of cardiac signals and R$\mathrm{R}$ intervals. The process electrocardiogram digital image is divided into two, first the process of calculating the R-R intervals and second the process of extraction feature using Discrete Cosine Transform, followed by calculating the Euclidean Distance or City block Distance with normal electrocardiogram signal reference. Euclidean Distance results or City block Distance and R-R distance of electrocardiogram signals are then classified using Multiclass Support Vector Machine. The results of accuracy the classification four class that are cardiac normal, atrial premature beat arrhythmia, atrial flutter arrhythmia, and atrial fibrillation arrhythmia, are $81.9 \%$. The originality is used image to detect cardiac normal or cardiac arrhythmia by combined Discrete Cosine Transform, Euclidean distance or City block distance and Multiclass Support Vector Machine.
\end{abstract}

Keywords: ECG, Multiclass SVM, DCT, Euclidean distance, City block distance

\section{INTRODUCTION}

According to the World Health Organization the 10th largest cause of death in the world, 2018 [1] is caused by chronic not contagious diseases, with the first rank being cardiovascular disorders. This indicates that heart disease is one thing that needs to be treated more seriously. There have been many studies on cardiovascular disease, including the following.[2]in his research discussed the latest methods of neural evolutionary systems for the recognition of the health cardiac signals. One class cardiac signal is classified by machine learning algorithm (SVM, kNN, PNN, and RBFNN) and the percentage of the best classification results is by SVM method with an accuracy of $98.85 \%$. Machine Learning can be used also in nervous system [3].

[4]in the research here used digital signal processing to process electrocardiograph data. The electrocardiograph signal analyzed is a QRS signal using open source solution like Python programming language and the DSP library using $\mathrm{C}++$. Combined python and $\mathrm{C}++$ makes applications that are designed to have flexibility and efficiency, plus a graphical user interface that makes it easy to use applications.

[5]in his study discussed three types of heart rate detection algorithms from electrocardiographs. The algorithm is based on statistics and differential mathematical methods. All of these methods were compared with stress test measurements. The result of a very simple filter design allows this algorithm to be implemented on a microprocessor and can be implemented in real-time.

[6]conducted a study to develop a real-time system algorithm in the detection of complex QRS from electrocardiograph signals using MATLAB. Complex QRS detection is performed by analyzing slope, amplitude and signal width.

[7]in his research, ECG was obtained with BIOPAC MP 100. The signal was then recorded and filtered using a Low Pass FIR filter with a cutoff frequency of $40 \mathrm{~Hz}$. Feature extraction using Discrete Wavelet Transform and statistical parameters such as mean, variance, standard deviation, etc. The classification in this study uses Artificial Neural Networks (ANN). The identification system accuracy is $100 \%$. ANN can also applicated in control problems to increasing the performance [8].

[9]using multiclassification by utilizing wavelet thresholding and support vector machines. wavelet multiresolution analysis is used to eliminate interference at frequency beyond the frequency interval of ECG signals. By using WMRA, the frequency bands below $0.05 \mathrm{~Hz}$ and above $100 \mathrm{~Hz}$ should be excluded from an ECG signal. because these frequencies are not ECG frequencies. The results obtained have an accuracy of $95.23 \%$.

[10]made developments for portable electrocardiogram devices so that they were easily carried to other places with the intention that users could use them more efficiently and in real-time. The device can detect an R or QRS interval signal and can also warn users of the device. In this paper, the method used is the first derivative filter and the max filter to analyze ECG signals. The ECG signal is removed by the filter so the analysis can be better done. Data as testing was taken from MIT-BIH and the results obtained were $\mathrm{Se}=$ $99.80 \%,+\mathrm{P}=99.80 \%$, and $\mathrm{DER}=0.36 \%$. 
The heart consists of two muscle lumps that contracts and in order rhythmically, pumping blood throughout the body. This contraction starts from the atrial sinus node which acts as a natural pace-maker, and spreads through atrial muscle and then to ventricular muscle via conduction system[11]. The propagation of this electrical signal follows a pattern. As a result of this activity, an electric current is generated on the surface of the body, triggering variations in the electrical potential on the surface of the skin. These signals can be observed through the help of adequate electrodes and devices. The electrical potentials differ between the points marked by the electrodes on the skin is usually enhanced by the aid of instrumentation (operational) amplifiers with optical isolation. After that, the signal is sent to the highpass filter; and as a second step, proceed to the anti-aliasing lowpass filter. Finally, the results appear in an analog to digital converter. This graphic registration of the acquisition process is called an electrocardiogram (ECG).

ECG signals can be segmented into heart rates. Each heartbeat consists of five standard waves labelled with the letters $\mathrm{P}, \mathrm{Q}, \mathrm{R}, \mathrm{S}$ and T. These waves indicate depolarization and re-polarization of the heart muscle phase. In addition, there are inter-wave timings called PR, QRS, QT, ST segments. This interval is shown in Figure 1.

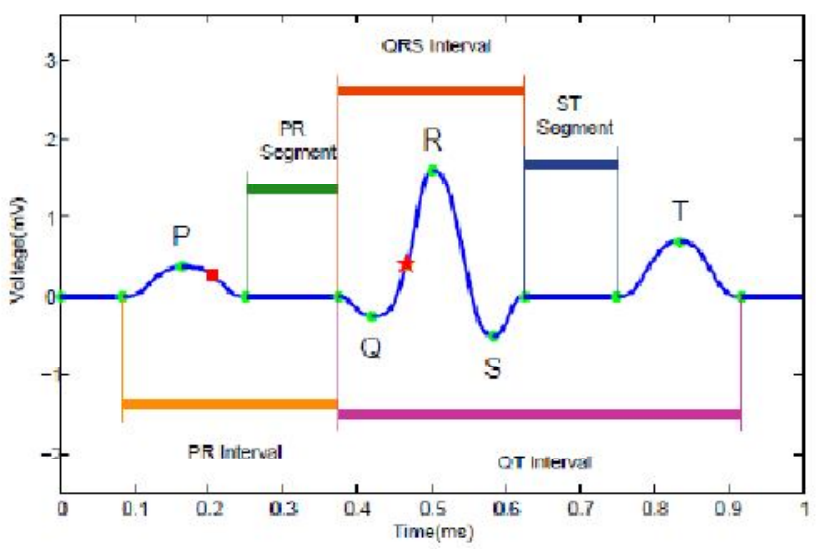

Figure 1: ECG curve.

Each heart contraction begins with a wave of excitement from the electrical activity of the activation of the SA node. From the SA node the wave will spread radially through the atria and get to the atrioventricular (AV) node and down the bundle branch to the ventricular muscle and Purkinje tissue. The SA node spontaneously depolarizes normally at a frequency of 60 to 100 times per minute. In the heart of a healthy person the SA node will automatically generate waves of stimulation of 60-100 beats per minute. Whereas in the heart of a person with atrial premature beat arrhythmia (Figure 2 (a)), the stimulus will occur ahead of the time they should, and in the heart of the person who has atrial flutter arrhythmia (Figure 2 (b)) the stimulus appears several times because of macro reentrant tachyarrhythmia and in the heart of the person experiencing atrial fibrillation arrhythmia (Figure 2 (c)) irregular stimulation. In this paper, we will classify normal people's cardiac signals, atrial premature beat arrhythmia (APB), atrial flutter arrhythmia (AFL), and atrial fibrillation arrhythmia (AFIB).

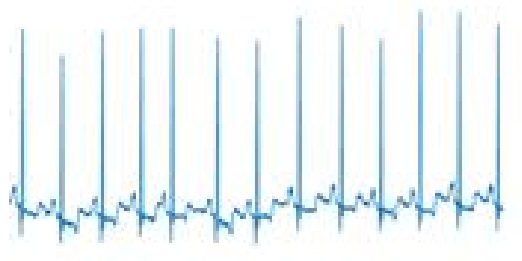

(a)

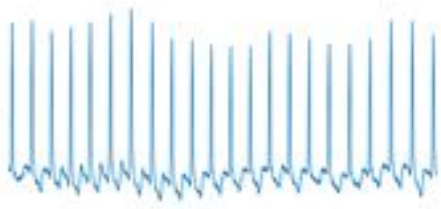

(b)

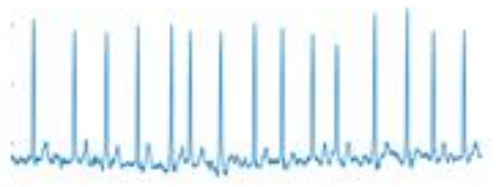

(c)

Figure 2: (a) Atrial Premature Beat, (b) Atrial Flutter, (c) Atrial Fibrillation.

\section{THEORETICAL BASIS}

2.1 Discrete Cosine Transform (DCT)

Discrete Cosine Transform (DCT)[12][13] is a transformators of digital image intensity information in the spatial domain into digital image data information in the frequency domain, whereas for converting digital image data information back in the frequency domain to digital image intensity information in the spatial domain IDCT (Inverse DCT) is used. Digital image is a two-dimensional data (image width and height), so that in the DCT transformation process two-dimensional DCT is used, and is performed per $N x N$-sized block, see Figure 3.

Two-dimensional DCT formula with block size (matrix) $\mathrm{N} \mathrm{x}$ $\mathrm{N}$ pixels [14]:

$D C T(i, j)=$
$\frac{1}{\sqrt{2 N}} C(i) C(j) \sum_{k=0}^{N-1} \sum_{l=0}^{N-1} \operatorname{pixel}(k, l) \cos \left\{\frac{[2 k+1] i \pi}{2 N}\right\} \cos \left\{\frac{[2 l+1] j \pi}{2 N}\right\}$

Where:

$C(p)=\frac{1}{\sqrt{2}}$ if $\mathrm{p}=0$, other than that 1 for $\mathrm{p}>0$

$i, j$ is integer $(0,1,2, \ldots, N-1)$; pixel $(k, l)$ is intensity of image pixel at positions $k, l ; k, l$ is coordinates of the pixel position of the image $(0,1,2, \ldots, N-1) ; D C T(i, j)$ is spectral value at position $i, j$.

The two-dimensional DCT formula $(\mathrm{N} \times \mathrm{N})$ above is often written in the form of a matrix (unitary) and is known as the cosine transform matrix $\mathrm{C}$ and is expressed in the form: 
Ratnadewiet al., International Journal of Emerging Trends in Engineering Research, 8(9), September 2020,5400 - 5407

where

$$
c(i, j)= \begin{cases}\frac{1}{\sqrt{N}} & \text { if } i=0 \\ \sqrt{\frac{2}{N}} \cos \left[\frac{(2 j+1) i \pi}{2 N}\right] & \text { if } i>0\end{cases}
$$

$c(i, j)$ is cosine matrix element at position $i, j ; i, j$ is integer $(0,1,2,3, . ., N-1) ; N$ is amount of data.

The properties of the cosine transformation matrix (DCT):

The cosine transformation matrix is real and orthogonal, so it satisfies:

where

$$
C=C^{*} \text { and } C^{-1}=C^{T}
$$

$C^{*}$ is matrix conjugate, $C^{-1}$ is matrix invers, $C^{T}$ is matrix transpose.

If pixels is an input matrix, then from Eq. 3 we get:

$$
D C T=C \text { Pixels } C^{T}
$$

and vice versa if the DCT value is known, then to obtain the Pixels value calculated through the equation:

$$
\text { Pixels }=C D C T C^{T}
$$

DCT, $C$, Pixels, $C^{T}$ is a matrix $N \times N$ Selection of significant values
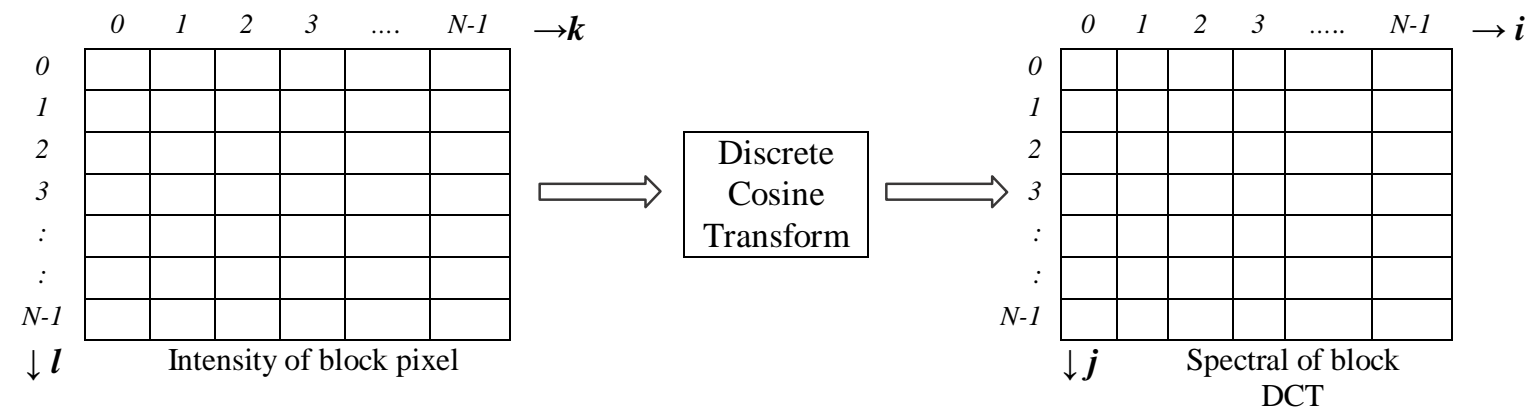

Figure 3:Transformation Block pixel.

Result the experiment from the [15], a typical set of images was transformed with DCT, apparently tending to produce large variance coefficients by forming a fixed variance contours, see Figure 4. The largest coefficient of variance tends to be in the upper left coordinate area.

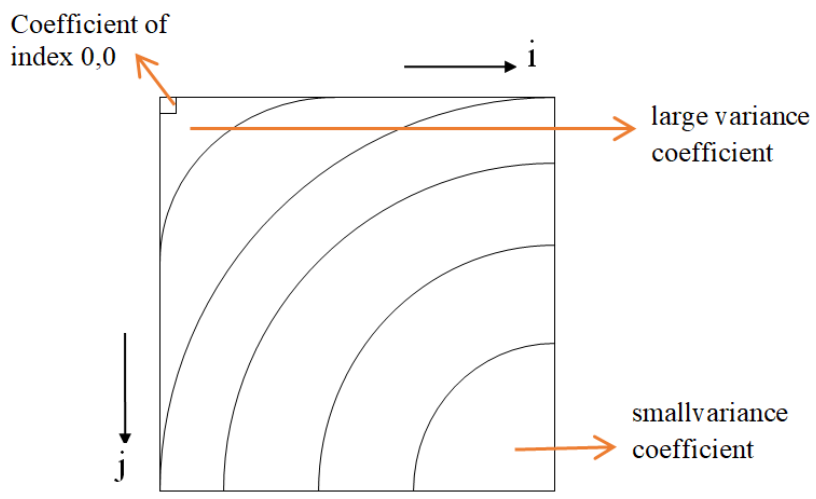

Figure 4: .Contours of the coefficient of variance in typical image transformation.

Large coefficient of variance indicates that the energy value of the stored data information is large which means also that significant image data information is stored a lot, generally to collect the information energy stored large data (significant value) is done by zigzag scanning method zonal mask on the area top left of each data block, see Figure 5.

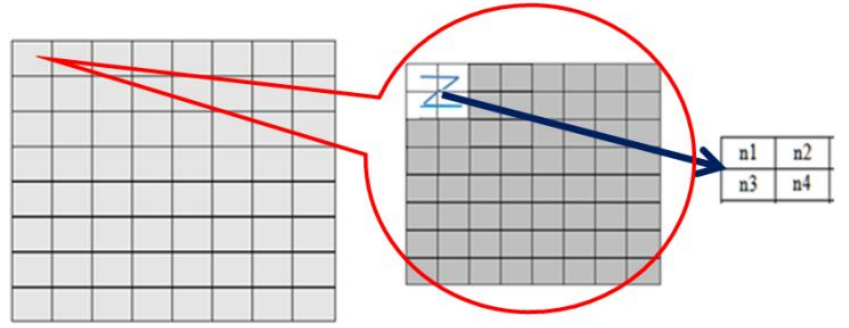

(a)

(b)

(c)

Figure 4: .(a) Image (b) zigzag scanning Block Image (c) taking significant value at zonal mask.

2.2 Euclidean distance (ED) or City Block Distance (CB) To measure the distance or similarity of two vectors $X=\left(x_{1}, x_{2}, \ldots, x_{n}\right)$ and $\quad Y=\left(y_{1}, y_{2}, \ldots, y_{n}\right)$ then the Euclidean distance (ED) between $X$ and $Y[16]$ is:

$$
E D=\sqrt{\sum_{i=1}^{n}\left|X_{i}-Y_{i}\right|^{2}}
$$

Whereas with the City block distance $(C B)$ the similarities of the two vectors $\mathrm{X}$ and $\mathrm{Y}[16]$ are:

$$
C B=\sum_{i=1}^{n}\left|X_{i}-Y_{i}\right|
$$

\subsection{Support Vector Machines (SVM)}

Support Vector Machine (SVM) has a concept of finding hyper plane (can refer to points, straight lines, twodimensional flat planes, and other fields with high dimensions) that separates data sets into two linear classes[17][18]. SVM will try to find the most optimum hyper plane, that is, if it is located right in the middle of the two classes so that it has the longest distance to the outer data in both classes (has a maximum margin).

SVM on linearly separable data is data that can be separated linearly. Suppose $\left(x_{1}, x_{2}, \ldots, x_{n}\right)$ is a dataset and 
$y_{i} \in\{+1,-1\}$ is the class label of data $x_{i}$. In the Figure 5 can be seen various alternative fields that can separate all data sets according to their class. However, the best separator field can not only separate data but also have the largest margins.

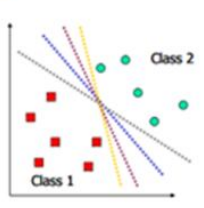

(a)

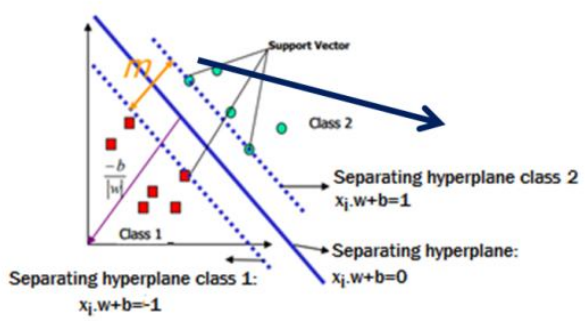

(b)
Figure 5: (a) alternative separating planes (b) best dividing planes with the largest margin $(\mathrm{m})$

The data in this boundary plane is called support vector. In the example in Figure 5(a), the two classes can be separated by a pair of parallel delimited fields. The first delimiter field restricts the first class while the second delimiter field restricts the second class, so that it is obtained (Figure 5(b)):

$$
\begin{aligned}
& x_{i} \cdot w+b \geq+1 \text { for } y_{i}=+1 \\
& x_{i} \cdot w+b \leq-1 \text { for } y_{i}=-1
\end{aligned}
$$

$w$ is the normal plane and $b$ is the position of the plane relative to the center coordinate. This margin value is maximized while still meeting Eq. (8) or Eq. (9).

\subsection{Multi Class SVM}

When SVM was first introduced by Vapnik, it could only classify data into two classes (binary classification). However, further research to develop SVM so that it can classify data that has more than two classes, continues to be done. There are two options for implementing multi-class SVM[19], namely by combining several binary SVMs or combining all data consisting of several classes into a form of optimization problem. However, in the second approach optimization problems that must be solved are far more complicated. The following are commonly used methods for implementing multi-class SVM with the following approaches:

Method "one -against-all"

This method compares one class with all other classes [20][21][9]. To classify data into $\mathrm{k}$ classes, a number of $\mathrm{k}$ binary SVM models must be built. Each i-binary SVM model is trained by using the entire training data, to find solutions to problems, namely by: where

$$
\min _{w^{m}, b^{i}, \xi^{i}}(w) \frac{1}{2}\left(w^{m}\right)^{T} w^{m}+C \sum_{i=1}^{l} \xi_{i}^{m}
$$

$$
\begin{aligned}
& \left(w^{m}\right)^{T} \phi\left(x_{m}\right)+b^{m} \geq 1-\xi_{i}^{m} \rightarrow y_{i}=m \\
& \left(w^{m}\right)^{T} \phi\left(x_{m}\right)+b^{m} \leq-1+\xi_{i}^{m} \rightarrow y_{i} \neq m \\
& \xi_{i}^{m} \geq 0, i=1, \ldots, l
\end{aligned}
$$

For examplesee Table 1, there are classification problems with 4 classes, so for training 4 binary SVMs are used as follows:
Table 1: Classification problem

\begin{tabular}{lll}
\hline $\mathbf{Y}_{\mathbf{i}}=\mathbf{1}$ & $\mathbf{Y}_{\mathbf{i}}=\mathbf{- 1}$ & Hypothesis \\
\hline Class 1 & Not Class & $f_{1}(x)$ \\
& 1 & $=\left(w_{1}\right) x+b_{1}$ \\
Class 2 & Not Class & $f_{2}(x)$ \\
& 2 & $=\left(w_{2}\right) x+b_{2}$ \\
Class 3 & Not Class & $f_{3}(x)$ \\
& 3 & $=\left(w_{3}\right) x+b_{3}$ \\
Class 4 & Not Class & $f_{4}(x)$ \\
& 4 & $=\left(w_{4}\right) x+b_{4}$ \\
\hline
\end{tabular}

Method "one $\neg$ against $\neg$ one"

Using this method, $[k(k-1) / 2]$ is built into a binary classification model ( $k$ is the number of classes). Each classification model is trained on data from two classes.

Method Directed Acyclic Graph Support Vector Machine (DAGSVM)

Training using this method is the same as the one-againstone method, namely by constructing $[k(k-1) / 2]$ binary SVM classification models. However, when testing binary directed acyclic graphs.

\subsection{Evaluation}

Evaluation [2][22] is done by calculating the accuracy value (AC) Eq.14, sensitivity value (SE) Eq.15, and specificity value (SP) Eq.16with the following equation:

$$
\begin{aligned}
A C & =\left(\sum_{i=1}^{N} \frac{T P+T N}{T P+F P+T N+F N}\right) \cdot 100 \% \\
S E & =\left(\sum_{i=1}^{N} \frac{T P}{T P+F N}\right) \cdot 100 \% \\
S P & =\left(\sum_{i=1}^{N} \frac{T N}{F P+T N}\right) \cdot 100 \%
\end{aligned}
$$

where

$N$ is number of set; $T P$ is True Positive corresponds to the number of times that the classifier correctly predicts a heartbeat without arrhythmia; $T N$ is True Negative quantifies the number of heart beats with arrhythmia that are predicted correctly; FP is False Positive gives the number of arrhythmia heartbeats classified as normal; $F N$ is False Negative indicates the total of normal beats misclassified as arrhythmia.

\section{IMPLEMENTATION AND ANALYSIS}

ECG database was taken from MIT-BIH Arrhythmia in 4 classes[23], namely 30 normal sinus rhythm, 12 atrial premature beats, 20 atrial flutter, and 10 atrial fibrillation. One image represents 10 seconds (3600 samples) fragments of the ECG signal were randomly selected. Only one signal lead is used, namely MLII.

The normal cardiac signal classification system and arrhythmias can be described as Figure 6. In training, the system input is the image of the cardiac signal which is then transformed into DCT, then feature extraction is taking 4 transformation values per block of $8 \times 8$ pixels, followed by zigzag scanning so that the data becomes vector. The average vector from a set of training images will be a reference vector in the process of calculating Euclidean distance or City block distance.

In the testing process there are two processes: first the same thing as the training process is carried out on the test image, the second is sought for the peak R for the signal that 
is the largest value that is more than the threshold value for each one curve, see Figure 7 , then the pixel position of all peak $\mathrm{R}$ and look for RR interval values are calculated by calculating the difference between the peak position $R$ and the value of the peak position $\mathrm{R}$ next to it. After all $\mathrm{R}-\mathrm{R}$ intervals have been obtained, a minimum $\mathrm{R}-\mathrm{R}$ interval value representing the image is selected The $R-R$ interval results are then combined with Euclidean distance or City block distance and are classified with Multiclass SVM.

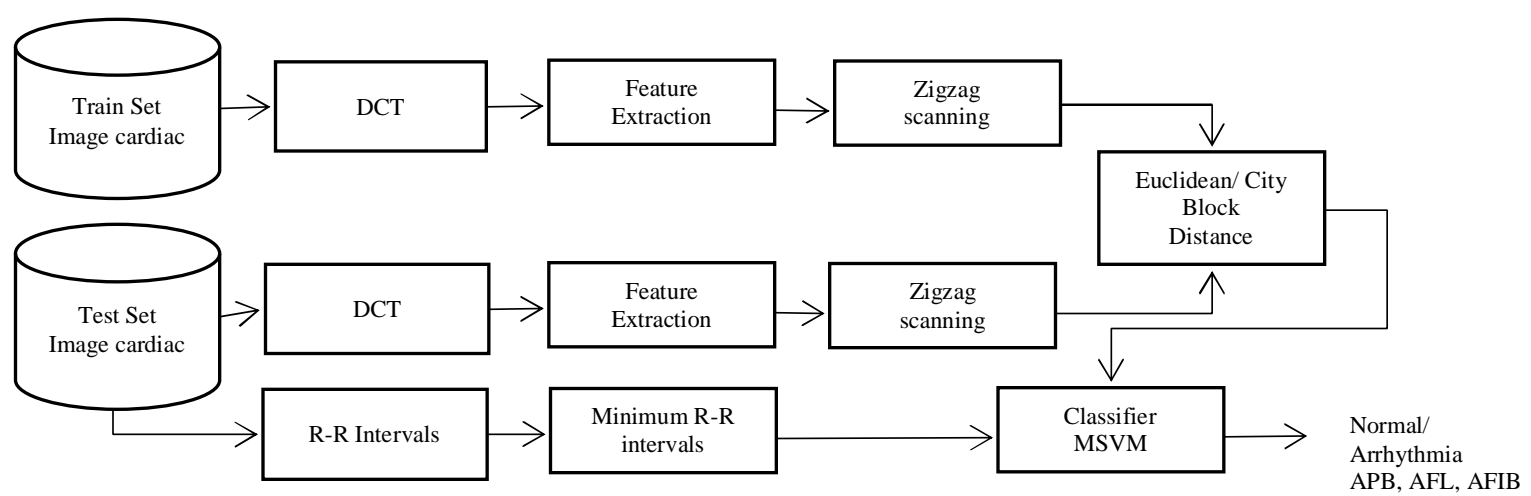

Figure 6: System for classifying normal cardiac signals and arrhythmias

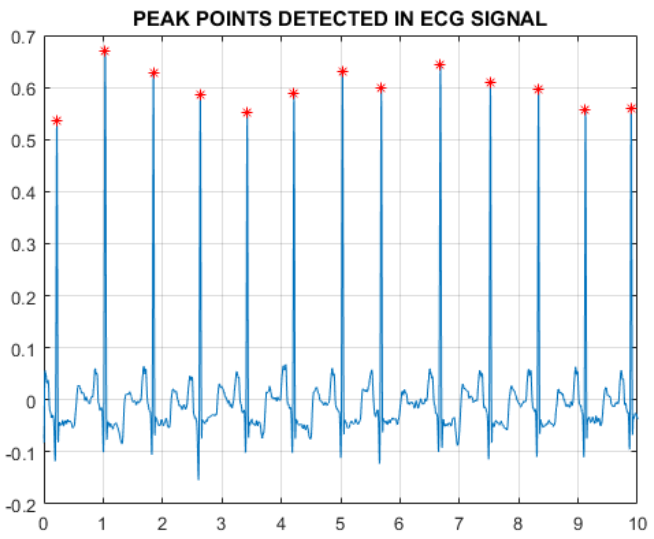

Figure 7:. Results of peak digital data detection of an ECG signal from MIT

Euclidean distance versus R-R intervals shows in Figure 8 and city block distance versus RR intervals shows in Figure 9, with Quadratic SVM 4-fold with accuracy $81.9 \%$. Normal cardiac data as class 0 are colored blue, cardiac atrial premature beat arrhythmia data as class 1 are colored red, cardiac atrial flutter arrhythmia data as class 2 are colored yellow, and cardiac atrial fibrillation arrhythmia data as class 3 are colored purple. The scatter plot shows that the classifications of the four classes are already separated by using the extraction of the DCT and Euclidean distance or city block distance features.

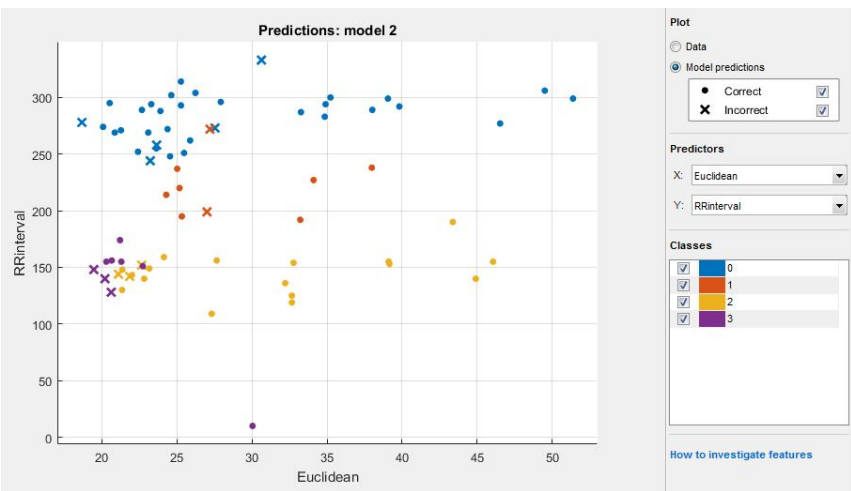

Figure 8: Euclidean distance versus R-R intervals with accuracy $81.9 \%$

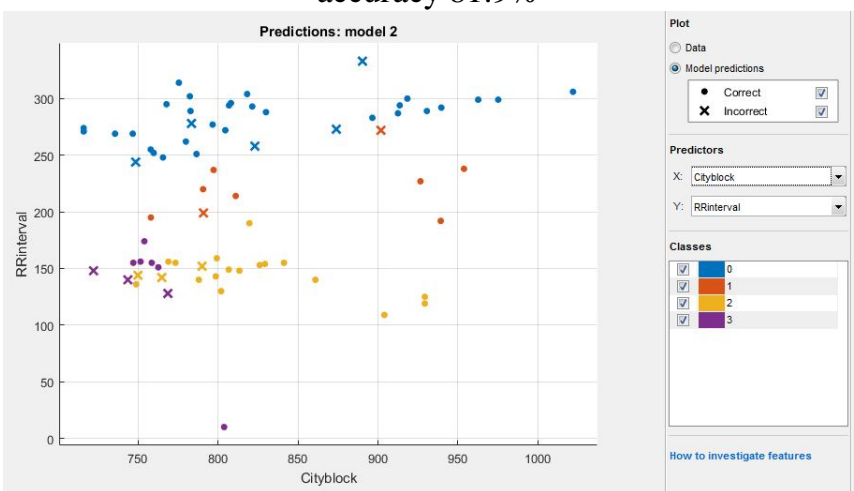

Figure 9: City block distance versus R-R intervals with accuracy $81.9 \%$

Figure 10 shows the vertical axis confusion matrix representing the vertical axis representing the true class and the horizontal axis representing the predicted class, while the values in the matrix represent the amount of data associated with the true class and class prediction. The green matrix states that the data is correct, while the pink color indicates the false prediction. The values on the vertical and horizontal axes of 0 represent normal classes, 1 represent atrial premature beat arrhythmia classes, 2 represent atrial 
flutter arrhythmia classes, and 3 represent atrial fibrillation arrhythmia classes.

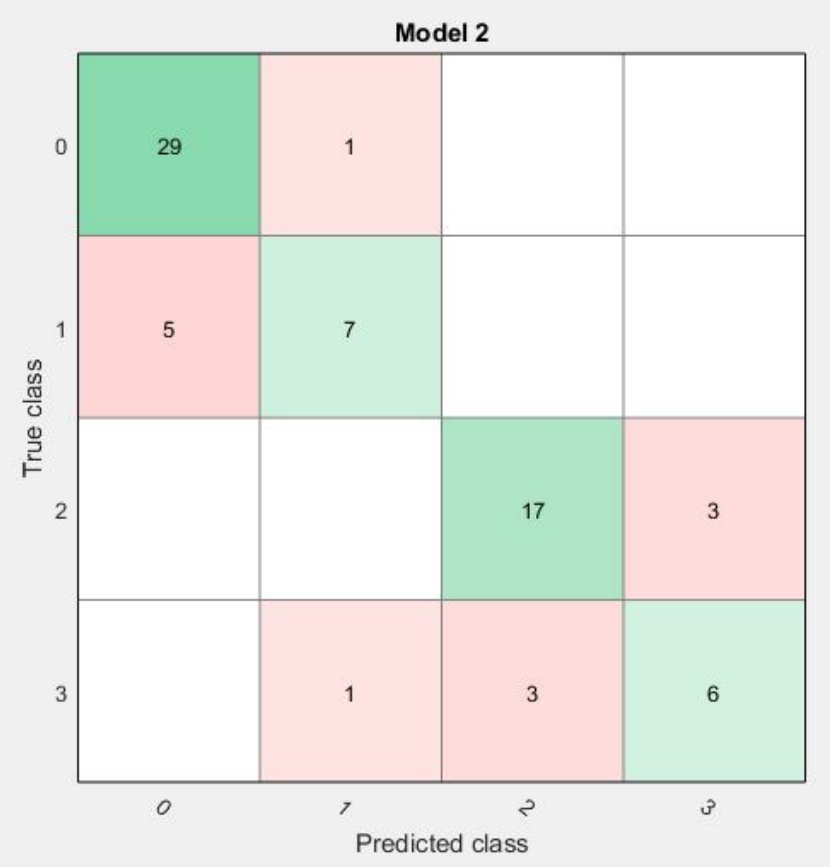

Figure 10: Number of observation every class.

In the first row Figure 10 of 30 normal cardiac data which is classified as true class (class 0) predicted 29 as normal data (class 0) and 1 data as cardiac atrial premature beat arrhythmia (class 1 ). So that the data is correct in class 0 and predicted that there is $97 \%$ in class 0 (correct prediction) and in class 0 data that is predicted in class not 0 there is $3 \%$ (incorrect prediction).

In the second row Figure 10 of 12 cardiac atrial premature beat arrhythmia data which are classified as true class (class 1) predicted 5 as normal data (class 0) and 7 data as atrial premature beat arrhythmia (class 1). So that the data is correct in class 1 and predicted by class 1 there are $58 \%$ (true prediction) and class 1 data that is predicted in class not 1 is $42 \%$ (incorrect prediction).

In the third row Figure 10 of 20 atrial flutter arrhythmia cardiac data which is included in true class (class 2) predicted 17 as atrial flutter arrhythmia data (class 2) and
3 data as atrial fibrillation arrhythmia (class 3 ). So that the data is correct in class 2 and predicted by class 2 there are $85 \%$ (correct prediction) and in class 2 data that is predicted in class not 2 there is $15 \%$ (incorrect prediction).

In the fourth row Figure 10 of 10 atrial fibrillation arrhythmia cardiac data which is classified as true class (class 3) predicted 1 as atrial premature beat arrhythmia data (class 1), 3 as atrial flutter arrhythmia data (class 2), and 6 data as atrial fibrillation arrhythmia (class 3 ). So that the data is correct in class 3 and $60 \%$ predicted by class 3 (correct prediction) and in class 2 data predicted in class not 2 there are $40 \%$ (incorrect prediction).

Now we count $T P, T N, F N$, and $F P$ for overall system from confusion matrix,

$$
\begin{gathered}
T P=29+7+17+6=59 \\
T N=29+7+17+6=59 \\
F N=1+5+3+(1+3)=13 \\
F P=5+(1+1)+3+3=13
\end{gathered}
$$

Accuracy for overall system is

$$
\begin{gathered}
A C=\left(\sum_{i=1}^{N} \frac{T P+T N}{T P+F P+T N+F N}\right) \cdot 100 \% \\
=\frac{59+59}{59+13+59+13} \cdot 100 \%=81.9 \%
\end{gathered}
$$

Sensitivity for overall system is

$$
\begin{aligned}
S E= & \left(\sum_{i=1}^{N} \frac{T P}{T P+F N}\right) \cdot 100 \%=\frac{59}{59+13} \cdot 100 \%=81.9 \% \\
& \text { Specificityfor overall system is } \\
S P= & \left(\sum_{i=1}^{N} \frac{T N}{F P+T N}\right) \cdot 100 \%=\frac{59}{13+59} \cdot 100 \%=81.9 \%
\end{aligned}
$$

Table 2 shows accuracy, sensitivity, and specificity for each class, for overall $81.9 \%$, and for each class can be seen on Table 2.

Figure 11 shows the percentage of True positive rate and False negative rate for each class. The results of the percentage of True positive rate are greater than the percentage of False negative rate which means the number of correct predictions is more than the false prediction.

Table 2:The result accuracy, sensitivity, specificity

\begin{tabular}{llllllll} 
& TP & TN & FN & FP & AC & SE & SP \\
\hline Normal & 29 & 29 & 1 & 5 & $90.6 \%$ & $96.7 \%$ & $85.3 \%$ \\
Atrial premature beat & 7 & 7 & 5 & 2 & $66.7 \%$ & $58.3 \%$ & $77.8 \%$ \\
arrhythmia, & 17 & 17 & 3 & 3 & $85.0 \%$ & $85.0 \%$ & $85.0 \%$ \\
Atrial flutter arrhythmia, & 6 & 6 & 4 & 3 & $63.2 \%$ & $60.0 \%$ & $66.7 \%$ \\
Atrial fibrillation arrhythmia & 59 & 59 & 13 & 13 & $81.9 \%$ & $81.9 \%$ & $81.9 \%$ \\
Overall & & & & & & & \\
\hline
\end{tabular}




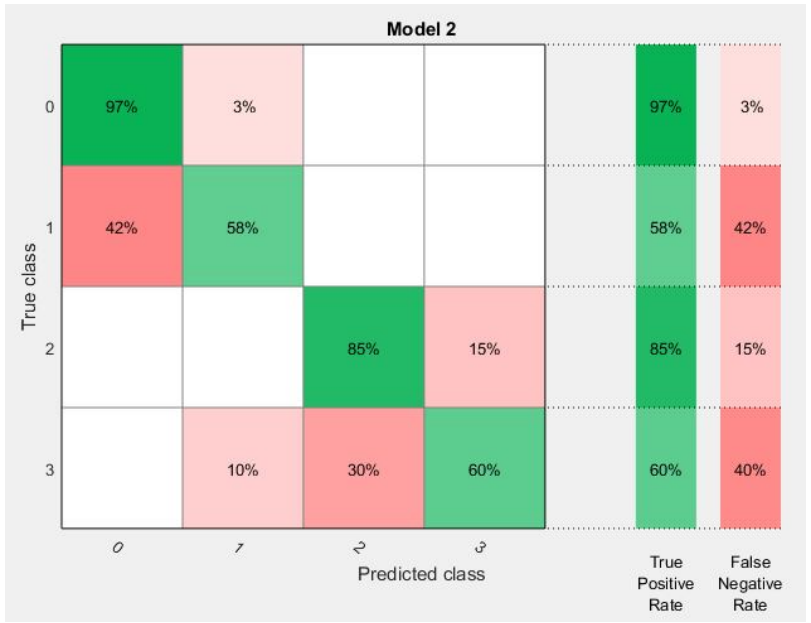

Figure 11:True positive rate and False negative rate

Figure 12 shows the percentage of Positive predictive value and False discovery rate for each class. Prediction of Positive predictive value percentage is greater than the percentage of False discovery rate, the system is good.InFigure 12, the percentage of positive prediction value based on column readings, if prediction is class 0 and true data class 0 so prediction is correct. The correct prediction is $85 \%$ (true prediction), while prediction class 0 but the true data not class 0 so predictions is wrong. The wrong prediction is $15 \%$ (false prediction). The same method is done in columns 1, 2, and 3 and the prediction results can be seen in Figure 12.

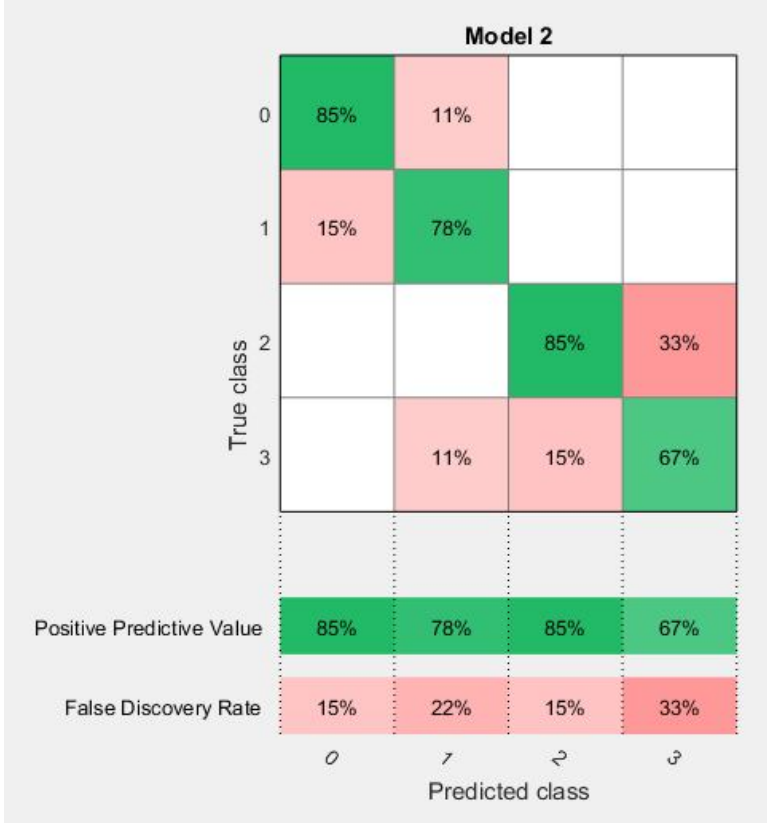

Figure 12:Positive predictive value and False discovery rate

\section{CONCLUSION}

The conclusions from this study are systems with feature extraction of DCT and Euclidean distance or Cityblock distance, and SVM multiclass classification to classify cardiac normal, atrial premature beat arrhythmia, atrial flutter arrhythmia, and atrial fibrillation arrhythmia have successfully predicted with an accuracy of $81.9 \%$, sensitivity of $81.9 \%$, and specificity of $81.9 \%$.

\section{ACKNOWLEDGEMENT}

Thank you so much for funding support that make this research possible to Universitas Kristen Maranatha.

\section{REFERENCES}

[1] W. H. Organization, "The top 10 causes of death," webpage, 2018. https://www.who.int/newsroom/fact-sheets/detail/the-top-10-causes-of-death.

[2] P. Pławiak, "Novel methodology of cardiac health recognition based on ECG signals and evolutionaryneural system," vol. 92, pp. 334-349, 2018, doi: 10.1016/j.eswa.2017.09.022.

[3] S. L. Dharmapuri, P. K. Dandamudi, V. M. Botcha, and B. P. Kolla, "Detecting central nervous system disorder using machine learning technique (XGB classifier)," Int. J. Emerg. Trends Eng. Res., vol. 8, no. 4, pp. 1142-1147, 2020, doi: 10.30534/ijeter/2020/31842020.

[4] W. Kosinski et al., "Digital Signal Processing in Ecg Recorder With Python-Based Software," J. Med. informatics Technol., vol. 6, no. September, p. IT-61 until IT-68, 2003.

[5] J. Parák and J. Havlík, "ECG signal processing and heart rate frequency detection methods," Proc. Tech. Comput. Prague, 2011, [Online]. Available: http://amber2.feld.cvut.cz/bmeg/wpcontent/uploads/2012/03/Parak-TCP-2011.pdf.

[6] P. Batra and R. Kapoor, "A Novel Algorithm for ECG Signal Processing,” vol. 8491, pp. 103-107, 2013.

[7] N. Yadav, M. Duhan, and A. Rose, "Biometric Human Recognition using ECG Signals," Int. Adv. Res. J. Sci. Eng. Technol., vol. 4, no. 6, pp. 168-171, 2017, doi: 10.17148/IARJSET.2017.4630.

[8] D. A. Petrosov, R. A. Vashchenko, A. A. Stepovoi, and N. V. Petrosova, "Application of artificial neural networks in genetic algorithm control problems," Int. J. Emerg. Trends Eng. Res., vol. 8, no. 1, pp. 177-181, 2020, doi: 10.30534/ijeter/2020/24812020.

[9] Q. Qin, J. Li, and C. Liu, "Multi-classification of cardiac diseases utilizing wavelet thresholding and support vector machine Multi-classification of Cardiac Diseases Utilizing Wavelet Thresholding and Support Vector Machine," in AIP Conference Proceedings, 2019, vol. 020026, no. January, pp. 15, doi: 10.1063/1.5085539.

[10] T. W. Bae and K. K. Kwon, "Efficient Real-Time R and QRS Detection Method Using a Pair of Derivative Filters and Max Filter for Portable ECG Device," Appl. Sci., vol. 9, no. 4128, 2019, doi: 10.3390/app9194128.

[11] M. G. Khan, Rapid ECG Interpretation. Humana Press, 2008.

[12] M. Nelson and J.-L. Gailly, The Data Compression Book. M\&T Books, 1996.

[13] M. S. Nixon and A. S. Aguado, Feature Extraction 
and Image Processing. London: Newnes, 2002.

[14] S. Dabbaghchian, M. P. Ghaemmaghami, and A. Aghagolzadeh, "Feature extraction using discrete cosine transform and discrimination power analysis with a face recognition technology," Pattern Recognit., vol. 43, no. 4, pp. 1431-1440, 2010, doi: 10.1016/j.patcog.2009.11.001.

[15] P. M. Embree and B. Kimball, C language algorithms for digital signal processing. PrenticeHall, 1991.

[16] S. Cha, "Comprehensive Survey on Distance / Similarity Measures between Probability Density Functions," Int. J. Math. Model. Methods Appl. Sci., vol. 1, no. 4, 2007.

[17] C. Shen, W. Kao, Y. Yang, M. Hsu, Y. Wu, and F. Lai, "Expert Systems with Applications Detection of cardiac arrhythmia in electrocardiograms using adaptive feature extraction and modified support vector machines," Expert Syst. Appl., vol. 39, no. 9, pp. 7845-7852, 2012, doi: 10.1016/j.eswa.2012.01.093.

[18] N. Cristianini and J. S. Taylor, An Introduction to Support Vector Machines and other kernel-based learning methods. Cambridge University Press, 2000.

[19] M. Hejazi et al., "Applied Artificial Intelligence : An Multiclass Support Vector Machines for Classification of ECG Data with Missing Values," no. August, 2015, doi: 10.1080/08839514.2015.1051887.

[20] Y. Liu and Y. F. Zheng, "Classification Reliability Multi-Class," in Proceedings of International Joint Conference on Neural Network, 2005, pp. 849-854.

[21] G. Frost, S. Jones, L. J., and Van Der Laan S., "A Survey of Sustainability Reporting Practices of Australian Reporting Entities," Aust. Account. Rev., vol. 15, no. 1, p. 89, 2005.

[22] L. B. Marinho, N. D. M. M. Nascimento, J. Wellington, and M. Souza, "A novel electrocardiogram feature extraction approach for cardiac arrhythmia classification," Futur. Gener. Comput. Syst., vol. 97, pp. 564-577, 2019, doi: 10.1016/j.future.2019.03.025.

[23] Physionet, "PhysioNet."

https://archive.physionet.org/cgi-bin/atm/ATM. 